\title{
Solution of system of the Fredholm integral equation of the first kind
}

\author{
Vladimir I. Uskov \\ Voronezh State of Forestry and Technologies named after G.F. Morozov, Voronezh, Russia \\ E-mail: vum1@yandex.ru
}

\begin{abstract}
The article is devoted to the study of a system of two inhomogeneous Fredholm integral equations of the first kind with two required functions depending on one variable. Integral equations describe the restoration of a blurred image, production costs, etc. Fredholm integral equations with one desired function have been considered in many works, but relatively few works have been devoted to systems of such equations. The questions of stability for the solution of systems and the construction of a regularizing system of equations were investigated, but the solution was not constructed in an explicit form. In this paper, the kernels depend on two variables. The case is considered: in the kernels and inhomogeneities, the variables are separated in the equations; these functions are decomposed on the basis of two functions on the interval of integration. Examples of basic functions are given. A condition is determined under which the system has a unique solution in the chosen basis, formulated as a theorem. The solution is found in the form of an expansion in this basis. To illustrate the results obtained, an example is considered
\end{abstract}

Keywords: system of Fredholm integral equations, first kind, basis of two functions, solution, uniqueness, formula, example. 


\title{
Решение системы интегральных уравнений Фредгольма первого рода
}

\author{
Владимир Игоревич Усков \\ Воронежский государственный лесотехнический университет имени Г.Ф. Морозова, \\ Воронеж, Россия \\ E-mail: vum1@yandex.ru
}

\begin{abstract}
Аннотация. Статья посвящена изучению системы двух неоднородных интегральных уравнений Фредгольма первого рода с двумя искомыми функциями, зависящими от одной переменной. Интегральными уравнениями описывается восстановление размытого изображения, издержки производства и т.д. Интегральные уравнения Фредгольма с одной искомой функцией рассматривались в многих работах, но системам таких уравнений посвящено сравнительно мало работ. Исследовались вопросы устойчивости для решения систем и построения регуляризирующей системы уравнений, но решение в явном виде не строилось. В настоящей работе ядра зависят от двух переменных. Рассматривается случай: в ядрах и неоднородностях в уравнениях разделены переменные; эти функции раскладываются по базису из двух функций на интервале интегрирования. Приводятся примеры базисных функций. Определено условие, при котором система имеет единственное решение в выбранном базисе, сформулированное в виде теоремы. Найдено решение в виде разложения по данному базису. Для иллюстрации полученных результатов рассматривается пример.
\end{abstract}

Ключевые слова: система интегральных уравнений Фредгольма, первый род, базис из двух функций, решение, единственность, формула, пример

\section{1. Введение}

Рассматривается система линейных интегральных уравнений Фредгольма первого рода:

$$
\begin{aligned}
& \int_{a}^{b} K_{11}(x, s) y_{1}(s) d s+\int_{a}^{b} K_{12}(x, s) y_{2}(s) d s=f_{1}(x), \\
& \int_{a}^{b} K_{21}(x, s) y_{1}(s) d s+\int_{a}^{b} K_{22}(x, s) y_{2}(s) d s=f_{2}(x),
\end{aligned}
$$

где заданы непрерывные по совокупности переменных в прямоугольнике $\Pi=X \times X$, где $X=$ $[a ; b]$, функции $K_{i j}(x, s)$, непрерывные функции $f_{i}(x)$ в $X, i, j=1,2$. Функции $K_{i j}(x, s)$ называются ядрами. 
Под решением системы (1), (2) подразумеваются функции $y_{i}(x), i=1,2$, непрерывные в $X$ и удовлетворяющие (1), (2) в $X$.

Актуальность темы связана с большим количеством приложений интегральных уравнений Фредгольма: ими описывается, например, восстановление размытого изображения [1], издержки производства [2] и т.д.

В работах [3], [4] (на конечном и бесконечном проиежутке интегрирования соответственно) для систем линейных интегральных уравнений Фредгольма построены регуляризирующие системы уравнений в пространстве интегрируемых с квадратом функций и получены оценки устойчивости для решений систем, но решение в явном виде не находилось.

Настоящая работа является логическим продолжением работы [5] автора этой статьи. Здесь все заданные функции полагаются разложенными по заданному базису из двух функций. Искомое решение системы находится также в виде разложения по этому базису. Приводится иллюстрирующий пример.

\section{2. О базисных функциях}

Пусть $H=\left\{h_{1}(x), h_{2}(x)\right\}$ - базис на множестве $Y$ и $W(x)$ - вронскиан, построенный с помощью этих функций [6]:

$$
W(x)=\operatorname{det}\left(\begin{array}{ll}
h_{1}(x) & h_{2}(x) \\
h_{1}^{\prime}(x) & h_{2}^{\prime}(x)
\end{array}\right)
$$

Лемма. Последовательность функций $H$ образует базис в $Y$ тогда и только тогда, когда $W(x) \neq 0$ при каждом $x \in Y$.

Пример. Например, следующие последовательности функций образуют базис на множестве $Y$ :
a) $h_{1}(x)=x$,
$h_{2}(x)=x^{2}, \quad Y=(0 ; 1) ;$
б) $h_{1}(x)=\sin x$
$h_{2}(x)=\cos x$
$Y=\mathbb{R} ;$
в) $h_{1}(x)=e^{a x}$
$h_{2}(x)=e^{b x}$
$Y=\mathbb{R}$,

где a,b - произвольные вещественные постоянные.

Проверим это для последовательности а). Действительно, в силу леммы, имеем:

$$
W(x)=\operatorname{det}\left(\begin{array}{cc}
x & x^{2} \\
1 & 2 x
\end{array}\right)=-x^{2} \neq 0, x \in Y .
$$

\section{3. Решение системы (1), (2)}

Перейдем к решению системы (1), (2).

Наложим следующее условие. 
Условие 1. Все заданные функции разложены по базису $H$ на множестве $\tilde{X}$ :

$$
\begin{gathered}
K_{i j}(x, s)=K_{i j}^{(1)}(s) h_{1}(x)+K_{i j}^{(2)}(s) h_{2}(x), \\
f_{i}(x)=f_{i}^{(1)} h_{1}(x)+f_{i}^{(2)} h_{2}(x), \quad i, j=1,2
\end{gathered}
$$

(здесь $f_{i}^{(1)}, f_{i}^{(2)}$ - постоянные).

Тогда подстановка (3) в (1) приводит к равенствам:

$$
\begin{gathered}
h_{1}(x) \int_{a}^{b} K_{11}^{(1)}(s) y_{1}(s) d s+h_{2}(x) \int_{a}^{b} K_{11}^{(2)}(s) y_{1}(s) d s+ \\
h_{1}(x) \int_{a}^{b} K_{12}^{(1)}(s) y_{2}(s) d s+h_{2}(x) \int_{a}^{b} K_{12}^{(2)}(s) y_{2}(s) d s=f_{1}^{(1)} h_{1}(x)+f_{1}^{(2)} h_{2}(x) .
\end{gathered}
$$

Приравняв коэффициенты при базисных функциях $H$, получим систему:

$$
\begin{aligned}
& \int_{a}^{b} K_{11}^{(1)}(s) y_{1}(s) d s+\int_{a}^{b} K_{12}^{(1)}(s) y_{2}(s) d s=f_{1}^{(1)}, \\
& \int_{a}^{b} K_{11}^{(2)}(s) y_{1}(s) d s+\int_{a}^{b} K_{12}^{(2)}(s) y_{2}(s) d s=f_{1}^{(2)} .
\end{aligned}
$$

Теперь, подставив (3) в (2), получим систему:

$$
\begin{gathered}
h_{1}(x) \int_{a}^{b} K_{21}^{(1)}(s) y_{1}(s) d s+h_{2}(x) \int_{a}^{b} K_{21}^{(2)}(s) y_{1}(s) d s+ \\
h_{1}(x) \int_{a}^{b} K_{22}^{(1)}(s) y_{2}(s) d s+h_{2}(x) \int_{a}^{b} K_{22}^{(2)}(s) y_{2}(s) d s=f_{2}^{(1)} h_{1}(x)+f_{2}^{(2)} h_{2}(x) .
\end{gathered}
$$

Приравнивание коэффициентов при базисных функциях приводит к системе:

$$
\begin{aligned}
& \int_{a}^{b} K_{21}^{(1)}(s) y_{1}(s) d s+\int_{a}^{b} K_{22}^{(1)}(s) y_{2}(s) d s=f_{2}^{(1)}, \\
& \int_{a}^{b} K_{21}^{(2)}(s) y_{1}(s) d s+\int_{a}^{b} K_{22}^{(2)}(s) y_{2}(s) d s=f_{2}^{(2)} .
\end{aligned}
$$

Далее, рассматривается система (4), (5). Разложим искомые функции по базису $H$ : 


$$
y_{i}(x)=y_{i}^{(1)} h_{1}(x)+y_{i}^{(2)} h_{2}(x), \quad i=1,2
$$

Постоянные $y_{i}^{(1)}, y_{i}^{(2)}$ надлежит вычислить. В обозначении:

$$
M_{i j k}^{(l)}=\int_{a}^{b} K_{i j}^{(l)}(s) h_{k}(s) d s
$$

подстановка (6) в (4), (5) влечет следующую систему с четырьмя неизвестными:

$$
\begin{aligned}
& M_{111}^{(1)} y_{1}^{(1)}+M_{112}^{(1)} y_{1}^{(2)}+M_{121}^{(1)} y_{2}^{(1)}+M_{122}^{(1)} y_{2}^{(2)}=f_{1}^{(1)}, \\
& M_{111}^{(2)} y_{1}^{(1)}+M_{112}^{(2)} y_{1}^{(2)}+M_{121}^{(2)} y_{2}^{(1)}+M_{122}^{(2)} y_{2}^{(2)}=f_{1}^{(2)}, \\
& M_{211}^{(1)} y_{1}^{(1)}+M_{212}^{(1)} y_{1}^{(2)}+M_{221}^{(1)} y_{2}^{(1)}+M_{222}^{(1)} y_{2}^{(2)}=f_{2}^{(1)}, \\
& M_{211}^{(2)} y_{1}^{(1)}+M_{212}^{(2)} y_{1}^{(2)}+M_{221}^{(2)} y_{2}^{(1)}+M_{222}^{(2)} y_{2}^{(2)}=f_{2}^{(2)} .
\end{aligned}
$$

Составим определитель $\Delta$, составленный из коэффициентов в левой части (8)

$$
\Delta=\operatorname{det}\left(\begin{array}{cccc}
M_{111}^{(1)} & M_{112}^{(1)} & M_{121}^{(1)} & M_{122}^{(1)} \\
M_{111}^{(2)} & M_{112}^{(2)} & M_{121}^{(2)} & M_{122}^{(2)} \\
M_{211}^{(1)} & M_{212}^{(1)} & M_{221}^{(1)} & M_{222}^{(1)} \\
M_{211}^{(2)} & M_{212}^{(2)} & M_{221}^{(2)} & M_{222}^{(2)}
\end{array}\right)
$$

и определители $\Delta_{i}^{(l)}$, полученные из $\Delta$ заменой столбцов из свободных членов $\left(f_{1}^{(1)} f_{1}^{(2)} f_{2}^{(1)} f_{2}^{(2)}\right)^{T}$ следующим образом: в $\Delta_{1}^{(1)}$ заменяется первый столбец, в $\Delta_{1}^{(2)}-$ второй столбец, в $\Delta_{2}^{(1)}-$ третий столбец, в $\Delta_{2}^{(2)}-$ четвертый столбец.

Наложим следующее условие.

\section{Условие 2.}

$$
\Delta \neq 0
$$

Тогда система (8) имеет единственное решение, определяемое по формулам [7]:

$$
y_{i}^{(j)}=\frac{\Delta_{i}^{(j)}}{\Delta}, \quad i, j=1,2 .
$$

Тем самым, получен следующий результат.

Теорема. Пусть выполнены условия 1, 2. Тогда система (1), (2) имеет единственное решение в базисе H, определяемое по формулам (6), (10), (8), (9).

\section{4. Пример}

Найти решение системы в базисе $x^{2}, x$ на $(0 ; 1)$ 


$$
\begin{aligned}
& \int_{0}^{1}\left(3 x^{2}+2 s x\right) y_{1}(s) d s+\int_{0}^{1}\left(2 x^{2}+3 s x\right) y_{2}(s) d s=x^{2}+2 x \\
& \int_{0}^{1}\left(x^{2}+3 s x\right) y_{1}(s) d s+\int_{0}^{1}\left(3 x^{2}+s x\right) y_{2}(s) d s=x^{2}+4 x
\end{aligned}
$$

В п.2 было установлено, что система функций

$$
h_{1}(x)=x^{2}, h_{2}(x)=x
$$

образует базис на $(0 ; 1)$.

Далее,

$$
\begin{gathered}
K_{11}(x, s)=3 x^{2}+2 s x, \quad K_{12}(x, s)=2 x^{2}+3 s x \\
K_{21}(x, s)=x^{2}+3 s x, \quad K_{12}(x, s)=3 x^{2}+s x \\
f_{1}(x)=x^{2}+2 x, \quad f_{2}(x)=x^{2}+4 x
\end{gathered}
$$

Применим результаты теоремы из п.3. Условие 1 имеет место. Проверим выполнение условия 2. Действительно, вычисления показывают, что

$$
\Delta=-49 / 5184 \neq 0
$$

Это означает, что решение системы (11) в данном базисе единственно. Оно определяется формулами

$$
y_{1}(x)=48 x^{2}-\frac{222}{7} x, \quad y_{2}(x)=-\frac{120}{7} x^{2}+12 x
$$

\section{Список литературы}

[1] Полянин,А.Д. Справочник по интегральным уравнениям / А.Д. Полянин, А.В. Манжиров. - М.: Физматлит, 2003. - 608 с.

[2] Спирина, М.С. Интегральные уравнения при моделировании издержек / М.С. Спирина, П.А. Спирин // Вестник Поволжского университета. Серия: Экономика. - 2015. - № 2 (40). - C. 234-238.

[3] Асанов, А. Регуляризация и устойчивость систем линейных интегральных уравнений Фредгольма первого рода / А. Асанов, З.А. Каденова // Вестник Самарского государственного технического университета. Серия: Физико-математические науки. - 2005. - Вып. 38. - С. 11-14. 
[4] Орозмаматова, Ж.Ш. Регуляризация и оценки устойчивости решений систем линейных интегральных уравнений Фредгольма первого рода на оси / Ж.Ш. Орозмаматова // Международный научный журнал «Символ науки». - 2018. - № 1-2. - С. 12-18.

[5] Усков, В.И. Решение одного интегрального уравнения Фредгольма первого рода / В.И. Усков, В.И. Небольсина // Молодой ученый. - 2019. - № 40 (278). - С. 1-3.

[6] Понтрягин, Л.С. Обыкновенные дифференциальные уравнения / Л.С. Понтрягин. - М.: Наука, 1974. - $331 \mathrm{c}$.

[7] Гельфанд, И.М. Лекции по линейной алгебре / И.М. Гельфанд. - М.: Добросвет, МЦНМО, 1998. - $320 \mathrm{c}$. 